\title{
p.R270X MECP2 mutation and mortality in Rett syndrome
}

\author{
Le Jian ${ }^{1}$, Hayley L Archer ${ }^{2}$, David Ravine ${ }^{3}$, Alison Kerr ${ }^{4}$, Nick de Klerk ${ }^{1}$, \\ John Christodoulou ${ }^{5,6}$, Mark ES Bailey ${ }^{7}$, Crystal Laurvick ${ }^{1}$ and Helen Leonard ${ }^{*, 1}$
}

\begin{abstract}
${ }^{1}$ Telethon Institute for Child Health Research, Centre for Child Health Research, The University of Western Australia, Perth, Western Australia, Australia; ${ }^{2}$ Institute of Medical Genetics, Cardiff University, Heath Park, Cardiff, UK; ${ }^{3}$ Western Australian Institute of Medical Research and Centre for Medical Research, The University of Western Australia, Perth, Western Australia, Australia; ${ }^{4}$ Department of Psychological Medicine, University of Glasgow, Glasgow, UK; ${ }^{5}$ Discipline of Paediatrics and Child Health, University of Sydney, New South Wales, Australia; ${ }^{6}$ Western Sydney Genetics Program, Children's Hospital at Westmead, Sydney, Australia; ${ }^{7}$ Division of Molecular Genetics, Institute of Biomedical and Life Sciences, University of Glasgow, Glasgow, UK
\end{abstract}

Among cases in the Australian Rett Syndrome Database, the nonsense mutation p.R270X is one of the most commonly occurring single pathogenic MECP2 mutations. In two recent published reports of the MECP2 mutational spectrum the p.R270X appeared to be under represented. We hypothesised that increased mortality arising from this mutation may underlie this apparent discrepancy. We investigated our hypothesis in two independent study groups from Australia and the UK with prospective data collections (total $n=524$ ). Only females with Rett syndrome and an identified MECP2 mutation were included.

Significant differences in survival were detected among Rett syndrome cases grouped for the eight most frequent mutations (log-rank $\chi^{2}(7)=15.71, P=0.03$ ). Moreover, survival among cases with $\mathrm{p} . \mathrm{R} 270 \mathrm{X}$, when compared with survival among cases with all the other mutations was reduced (log-rank $\chi^{2}(2)=6.94$, $P=0.01)$. Our observation of a reduced survival associated with the p.R270X mutation offers an explanation for the under representation of p.R270X in older subjects with Rett syndrome. European Journal of Human Genetics (2005) 13, 1235-1238. doi:10.1038/sj.ejhg.5201479; published online 3 August 2005

Keywords: Rett syndrome; p.R270X; mortality; MECP2 mutations

\section{Introduction}

Rett syndrome was first reported in 1966 by the Austrian neurologist Andreas Rett and received worldwide recognition following a publication by Dr Bengt Hagberg et al in 1983. In 1999, the laboratory of Dr Huda Zoghbi ${ }^{2}$ identified mutations causative of Rett syndrome in the X-linked MECP2 gene, which encodes methyl-CpG-bind-

*Correspondence: Dr H Leonard, Centre for Child Health Research, The University of Western Australia, Telethon Institute for Child Health Research, PO Box 855, West Perth 6872, Subiaco, Western Australia, 6008. Tel: +6189489 7790; Fax: +61 894897700 ;

E-mail: hleonard@ichr.uwa.edu.au

Received 26 April 2005; revised 8 June 2005; accepted 30 June 2005; published online 3 August 2005 ing protein 2 (MeCP2). MECP2 mutations are now being detected in up to $80 \%$ of sporadic cases of typical Rett syndrome. ${ }^{3,4}$ p.R270X, a nonsense mutation located in the nuclear localisation signal (NLS) region, which is within the transcription repression domain (TRD), is among the most frequent of the recurrent mutations detected. ${ }^{5}$ Currently, it represents $9.6 \%$ of the pathogenic mutations recorded in RettBase. ${ }^{6}$ In recently published papers by Schanen et $\mathrm{al}^{7}$ and Smeets et $\mathrm{al}^{8}$ the proportions of patients with the p.R270X mutation were much lower $(2.4 \%(2 / 85)$ and $0 \%(0 / 24))$ than the $10.9 \%(11 / 101)\left(\chi^{2}=5.9, P=0.02\right)$ in the Australian population-based juvenile sample ${ }^{4}$ where, as in the British Isles Rett Survey (BIRS), ${ }^{9}$ it was the third most common mutation. The age range of the cases in 
these two recent reports was 19 months to 60 years with a high proportion of older children and adults represented in both. We hypothesised that the reduced prevalence of p.R270X among the older subjects could be reflecting a higher mortality associated with this mutation. Using longitudinal data (collected over the past 12 years in Australia and 22 years in the UK) on cases in whom mutations have been characterised, we have investigated whether p.R270X, which we have already shown to be a more severe mutation, ${ }^{4}$ is associated with increased mortality.

\section{Methods}

\section{Case recruitment}

Australian subjects The Australian Rett Syndrome Database (ARSD, www.ichr.uwa.edu.au/rett/aussierett/), established in 1993, is a population-based register with ongoing ascertainment of Rett syndrome cases born since $1976 .^{10}$ By early 2005, 278 individuals with verified Rett syndrome (23 of whom were known to have died) were registered with the ARSD. Among these, a pathogenic molecular abnormality has been identified in 175 (62.9\%). A further $60(21.6 \%)$ have been tested and no mutation found and $43(15.5 \%)$ have not been tested or no result is yet available. As would be expected, since mutation testing has only been available since $1999 / 2000$, a higher proportion of deceased cases, $8 / 43$ (18.6\%), were in the group that had not been tested, compared with a mortality to date of 9/175 (5.1\%) among those with a mutation and 6/60 (10.0\%) among those in whom genetic testing did not reveal a mutation. The current analysis excludes the one male with a MECP2 mutation and is restricted to those females (live and deceased) $(n=174)$ where a pathogenic mutation has been identified. The age range of this group was 2-29 years with a median of 14.5 years. X chromosome inactivation (XCI) status was evaluated by using the methods described previously. ${ }^{4}$ The pattern of XCI was classified as skewed when one $\mathrm{X}$ chromosome was active in $25 \%$ or less of the DNA sample tested, otherwise it was considered to be random. Using this technique, it was not possible to determine the linkage phase for XCI and $M E C P 2$. Informative results on XCI were available on $105 / 174$ cases, but in only three of the eight deceased.

To ensure that the analysis was limited to cases in whom prospectively collected data would be available, we restricted the Australian data to cases registered with ARSD. Thus, we did not include the 19 adult mutation positive cases of whom we were aware but who were not eligible for ARSD as they were born prior to 1976.

\section{UK subjects}

The BIRS is a UK database that has collected longitudinal data about people with Rett syndrome since 1982 and has received over 1200 notifications. ${ }^{9}$ Among cases enrolled in the survey who have died, mutational analysis had been arranged in 22/104 (21\%) with mutations detected in 15 $(68.2 \%)$. This study is restricted to the $296 \mathrm{UK}$ cases in the BIRS in whom a pathogenic MECP2 mutation has been identified, supplemented by a further 57 cases who had MECP 2 analysis in Cardiff $(n=353)$. After exclusion of male cases (as was also done with the Australian data as we felt the pattern of mortality in males was likely to be different), the final data set consisted of 350 female UK cases. Ages ranged from 1-54 years with a median of 11 years. Information on XCI was not available on the UK cases.

\section{Statistical analysis}

Survival status was obtained in slightly different ways according to the data source. For the ARSD there is ongoing contact with families of study participants and the study is generally notified at the time of death of any cases. Furthermore the Australian National Death Index ${ }^{11}$ enables detection of deaths among cases lost to follow-up. The BIRS also has ongoing contact with families and deaths are usually reported to AK. In addition, HA has contacted the clinicians of all patients who were tested in Cardiff, and this has resulted in further notifications of deaths. For cases notified to the BIRS, age at last clinical update as well as date of death were recorded. Any updated information available through genetic analysis at Cardiff was also included.

Overall survival from birth was determined using the Kaplan-Meier product-limit method. The log-rank test was applied to evaluate variations in survival among those in the different mutational groups. The eight most frequent recurrent $\mathrm{C}>\mathrm{T}$ transition mutations (p.T158M, p.R168X, p.R255X, p.R270X, p.R294X, p.R133C, p.R306C and p.R106W) were compared. Additionally, survival among cases with p.R270X was compared with survival in cases with all other mutations. Hazard ratios and 95\% CIs were estimated using Cox regression. In this investigation it was considered inappropriate to adjust for severity score as a covariate as it is likely to be on the causal pathway between mutation type and mortality.

\section{Results}

Among the 23 female deaths, eight were from ARSD, which represented $4.6 \%$ of the Australian cases while the 15 from the UK cohort represented $4.3 \%$ of British cases. Table 1 shows the distribution of cases by live/deceased status for the common recurrent mutations according to the data source. p.R270X was the individual mutation associated with most deaths $(n=5,11.4 \%)$. The other mutation with fewer cases but a high proportion of deaths was p.R106W where two of the 16 cases were deceased. Comparison of survival among the eight common mutations revealed a significant overall difference (log-rank $\chi^{2} \quad(7)=15.71$, $P=0.03)$. Moreover when survival of cases with $\mathrm{p} . \mathrm{R} 270 \mathrm{X}$ 
Table 1 The distribution of eight common and all other mutations by deceased status in Rett syndrome cases in Australia and the UK

\begin{tabular}{|c|c|c|c|c|c|c|}
\hline \multirow[b]{2}{*}{ Mutation } & \multirow[b]{2}{*}{ Age median (range) } & \multicolumn{2}{|c|}{ Australian cases } & \multicolumn{2}{|c|}{ UK cases } & \multirow[b]{2}{*}{ Total (\%) } \\
\hline & & Deceased & Alive & Deceased & Alive & \\
\hline p.R106W & $11.0(2.0-27.6)$ & 1 & 5 & 1 & 9 & $16(3.0)$ \\
\hline p.R133C & $15.2(4.0-41.0)$ & 0 & 10 & 0 & 24 & $34(6.5)$ \\
\hline p.T158M & $13.0(2.0-40.0)$ & 1 & 20 & 2 & 42 & $65(12.4)$ \\
\hline p.R168X & $10.9(2.0-42.0)$ & 0 & 20 & 1 & 37 & $58(11.1)$ \\
\hline p.R255X & $9.0(2.0-40.0)$ & 0 & 13 & 2 & 27 & $42(8.0)$ \\
\hline p.R270X & $12.6(2.0-30.0)$ & 4 & 10 & 1 & 29 & $44(8.4)$ \\
\hline p.R294X & $13.1(2.0-40.0)$ & 0 & 17 & 0 & 16 & $33(6.3)$ \\
\hline p.R306C & $17.0(3.0-39.0)$ & 1 & 10 & 0 & 19 & $30(5.7)$ \\
\hline Others & $12.0(1.0-54.0)$ & 1 & 61 & 8 & 132 & $202(38.6)$ \\
\hline All & & 8 & 166 & 15 & 335 & $524(100.0)$ \\
\hline
\end{tabular}

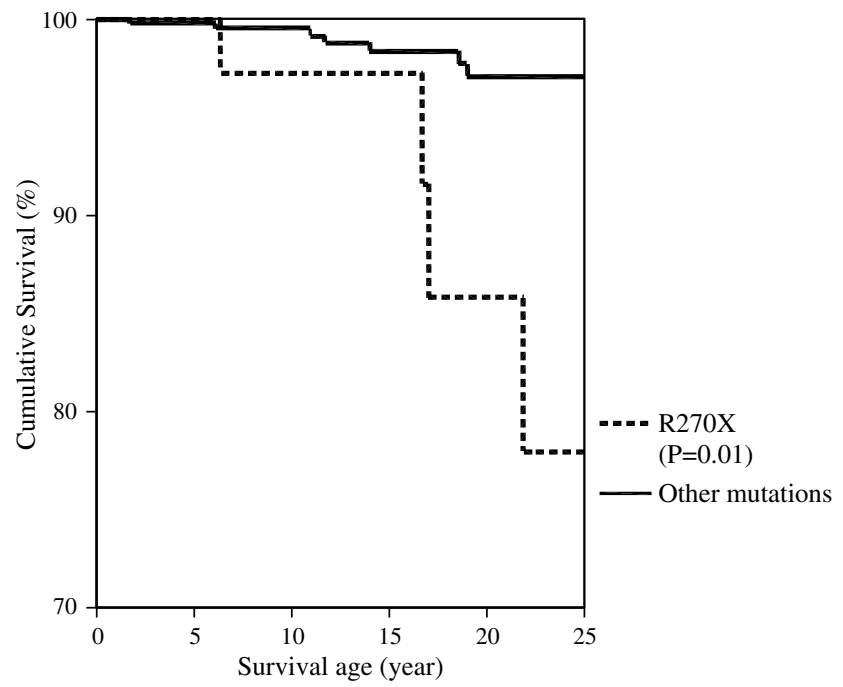

Figure 1 Survival with p.R270X mutation compared with other mutations.

was compared with those with all other mutations survival was clearly compromised (log-rank $\chi^{2}(2)=6.94, P=0.01$, see Figure 1 ) with a hazard ratio of 3.54 (CI 1.30-9.66). In the p.R270X survivors skewing was present in two of the nine $(22.2 \%)$ cases on whom data were available. This compares with a prevalence of skewing of $36.2 \%$ overall.

\section{Discussion}

Results from the first reported survival analysis of Rett syndrome cases in whom mutations have been characterised confirm our hypothesis that there is a higher mortality amongst cases arising from a p.R270X mutation. Strengths of the ARSD include the high ascertainment and follow-up of registered cases ${ }^{10,12}$ and the facility to update information on deaths from family and clinician notifications, as well as from linkage to the Australian National Death Index. However, although the ARSD provides population-based data, the underlying Australian population is only one third of that of the UK where the potential number of identifiable Rett syndrome cases is much greater. On the other hand, in the UK where there is also a mechanism for ascertainment and follow-up, and deaths are usually notified to $\mathrm{AK}$, it is possible that some deaths may have been missed. Moreover, because the majority of deceased cases, both in the Australian and UK registers, died before molecular testing first became available in 2000, the statistical power for this analysis remains low.

Until now research on mortality in Rett syndrome in general, particularly life expectancy projections, ${ }^{13}$ has been limited, both prior to and since the discovery of its genetic cause. $^{9,14,15}$ One population-based study in Western Sweden, which reported follow-up to the year 2000 of 54 females diagnosed between 1971 and 1998, included 10 deaths (18\%), which were usually sudden and unexpected. ${ }^{15}$ Because of the era of data collection, no mutational data were available for these cases. Although there is now a substantial body of research relating clinical severity to mutation type, ${ }^{4,16-18}$ none have explored the association between mutation type and mortality.

The findings of the present study are consistent with earlier results $4,9,16,19$ that have revealed a greater clinical severity among cases with mutations in the NLS region, p.R270X in particular. Our own earlier reports ${ }^{4,19}$ would have included information from some cases with a p.R270X mutation who have since died. The recently reported absence of p.R270X in the Belgian study of adolescents and adults with Rett syndrome ${ }^{8}$ and the low proportion in the US study ${ }^{7}$ compared with its prevalence in our prospectively ascertained cohort may well be reflecting a higher mortality associated with this mutation. 
There is some evidence to suggest that skewing of XCI may modulate the clinical severity (and hence possibly also mortality) of Rett syndrome. ${ }^{20,21}$ However, XCI data were not available for the UK cases nor for three of the four Australian p.R270X deaths and only two of the nine p.R270X survivors had a skewed pattern. Future $\mathrm{X}$-inactivation work, which will allow us to determine whether the normal or mutated X is inactivated, is required before we can fully investigate the impact of XCI on mortality.

In summary, our findings suggest that the p.R270X mutation, already known to be associated with more severe clinical features may also be associated with increased mortality. Questions about life expectancy are among the most commonly asked by families. ${ }^{22}$ Ongoing follow-up of national cohorts as well as international collaborations will be required to create the data to answer these questions and to gain insights into the genetic and environmental influences on mortality in this disorder. InterRett, ${ }^{23}$ which, with its international perspective, has already ascertained nearly 300 cases within 2 years, will offer further opportunities to generate the data needed to understand more of the complex relationship between genotype and phenotype in Rett syndrome.

\section{Acknowledgements}

We acknowledge the National Institute of Child Health and Human Development for its current funding of the Australian Rett Syndrome Database under NIH Grant number 1 R01 HD43100-01A1 PI and the Financial Markets Foundation for Children and the Rett Syndrome Australian Research Fund for previous funding. HL is funded by NHMRC program Grant 353514. JC is funded by NHMRC project Grants 185202 and 346603. HA is funded by The Health Foundation, $A K$ and $M B$ by the Rett Syndrome Association UK and MB by the Rett Syndrome Research Foundation, the International Rett Syndrome Association, the Rett Syndrome Association Scotland and the Scottish Hospital Endowments Research Trust. We express our gratitude to all the families who have participated in the study; the Australian Paediatric Surveillance Unit and the Rett Syndrome Association of Australia who facilitated case ascertainment in Australia. In addition, we thank the Rett Syndrome Association UK and the regional genetics and paediatrics services, which have all strongly supported Rett syndrome research in the United Kingdom.

\section{References}

1 Hagberg B, Aicardi J, Dias K, Ramos O: A progressive syndrome of autism, dementia, ataxia, and loss of purposeful hand use in girls: Rett's syndrome: report of 35 cases. Ann Neurol 1983; 14: $471-479$.

2 Amir RE, Van den Veyver IB, Wan M, Tran CQ, Francke U, Zoghbi HY: Rett syndrome is caused by mutations in X-linked MECP2, encoding methyl- CpG-binding protein 2. Nat Genet 1999; 23: $185-188$.

3 Renieri A, Meloni I, Longo I et al: Rett syndrome: the complex nature of a monogenic disease. J Mol Med 2003; 81: 346-354.

4 Colvin L, Leonard $\mathrm{H}$, de Klerk $\mathrm{N}$ et al: Refining the phenotype of common mutations in Rett syndrome. J Med Genet 2004; 41: $25-30$.

5 Dragich J, Houwink-Manville I, Schanen C: Rett syndrome: a surprising result of mutation in MECP2. Hum Mol Genet 2000; 9: $2365-2375$.

6 Christodoulou J: RettBASE: The IRSA MECP2 variation database; Sydney, Western Sydney Genetics Program, Wiley-Liss, Inc. http://mecp2.chw.edu.au/mecp2/,accessed 18 April 2005.

7 Schanen C, Houwink EJ, Dorrani N et al: Phenotypic manifestations of MECP2 mutations in classical and atypical Rett syndrome. Am J Med Genet 2004; 126A: 129-140.

8 Smeets E, Schollen E, Moog U et al: Rett syndrome in adolescent and adult females: clinical and molecular genetic findings. $A m \mathrm{~J}$ Med Genet 2003; 122A: 227-233.

9 Kerr AM, Prescott RJ: Predictive value of the early clinical signs in Rett disorder. Brain Dev 2005, in press.

10 Colvin L, Fyfe S, Leonard S et al: Describing the phenotype in Rett syndrome using a population database. Arch Dis Child 2003; 88: $38-43$.

11 Australian Institute of Health and Welfare. National Death Index (deaths from 1980 to present), AIHW: http://www.aihw.gov.au/ cancer/ndi/index.cfm,accessed 18 April 2005.

12 Leonard H, Bower C, English D: The prevalence and incidence of Rett syndrome in Australia. Eur Child Adolesc Psychiatr 1997; 6: $8-10$.

13 del Junco D, Nancy MN, Steve W et al: Survival in a large Cohort of US girls and Women with Rett Syndrome. J Child Neurol 1993; 8: $101-102$.

14 Kerr AM, Armstrong DD, Prescott RJ, Doyle D, Kearney DL: Rett syndrome: analysis of deaths in the British survey. Eur Child Adolesc Psychiatr 1997; 6 (Suppl 1): 71-74.

15 Hagberg B, Berg M, Steffenburg U: Three decades of sociomedical experiences from West Swedish Rett females 4-60 years of age. Brain Dev 2001; 23 (Suppl 1): S28-S31.

16 Huppke P, Held M, Handefeld F, Engel W, Laccone F: Influence of mutation type and location on phenotype in 123 patients with Rett Syndrome. Neuropediatrics 2002; 33: 63-68.

17 Hoffbuhr K, Devaney JM, LaFleur B et al: MeCP2 mutations in children with and without the phenotype of Rett syndrome. Neurology 2001; 56: 1486-1495.

18 Leonard H, Colvin L, Christodoulou J et al: Patients with the R133C mutation: is their phenotype different from patients with Rett syndrome with other mutations? J Med Genet 2003; 40: e52.

19 Leonard H, Moore H, Carey $\mathrm{M}$ et al: Genotype and early development in Rett syndrome: the value of international data. Brain Dev, in press.

20 Hoffbuhr KC, Moses LM, Jerdonek MA, Naidu S, Hoffman EP: Associations between meCP2 mutations, X-chromosome inactivation, and phenotype. Ment Retard Dev Disabil Res Rev 2002; 8: 99-105.

21 Moore H, Leonard H, de Klerk N et al: Health Service Use in Rett Syndrome. J Child Neurol 2004; 19: 42-50.

22 Hunter K: Looking from the inside out: a parent's perspective. Ment Retard Dev Disabil Res Rev 2002; 8: 77-81.

23 Moore H, Leonard H, Fyfe S, de Klerk N, Leonard N: InterRett the application of bioinformatics to international Rett syndrome research. Ann Hum Biol 2005; 32: 228-236. 